\title{
Implementing Overall Equipment Effectiveness in a Process Industry
}

\author{
V. Palanisamy ${ }^{1 *}$ and Jose Ananth Vino ${ }^{2}$ \\ 1,2Assistant. Professor, Department of Mechanical Engineering, Bharath University, Chennai-600 073; \\ vpsgce@gmail.com
}

\begin{abstract}
Overall equipment effectiveness (OEE) is a concept where the bottleneck operations of a particular process are reduced to certain extent. A pilot scale study is under taken in the product manufacturing industry and OEE concepts are implemented in the job floor. The three parameters such as availability, performance and quality of the process are taken up for this purpose. The OEE concepts were implemented in a periodic manner and continuous improvement in the job floor was monitored which proved some positive output.
\end{abstract}

Keywords: OEE, Availability, Performance, Quality.

\section{Introduction}

Overall equipment effectiveness (OEE) and Total effective equipment performance (TEEP) are closely related measurements that report the overall utilization of facilities, time and material for manufacturing operations. OEE quantifies how well a manufacturing unit performs relative to its designed capacity, during the periods when it is scheduled to run. TEEP measures OEE against calendar hours, i.e. 24 hours per day.

The main goal of the work is to obtain a best value to increase the production process in the casting company by using OEE.

We develop an IT integrated system to record the time intervals of OEE losses for the bottleneck equipment "wire bonder" in the semiconductor assembly industry. We integrate a communication system (that is developed by the wire bonder supplier) and MES (Manufacturing Execution System) into an ADC (Automated Data Collection) system for collecting useful data. The data quality is further guaranteed by the real time detection of equipment status from the communication system. The application of the ADC system has eliminated the unknown OEE losses from original $6 \%$ of manual recording system to near zero percent. Therefore, the UPH data can be collected automatically once a production lot (partition of a specific batch in an order) is finished. This resolves the accuracy and timeliness problem associated with traditional stopwatch sampling.

Wang T et al [1] found that Most semiconductor companies follow the SEMI E10 and E79 guidelines to measure equipment availability and efficiency via OEE. However, several problems need to be conquered before implementing OEE. For example, the time intervals of OEE losses are critical to the improvement studies, but it is very hard to collect reliable and accurate data. Therefore, people will find the unknown differences between the recorded losses from their operational system and OEE losses from SEMI E79 definition once people implement OEE. Furthermore, how to obtain the theoretical unit throughput or standard UPH (unit-per-hour machine rates) to determine the average processing rates of equipment is another issue to be conquered.

*Corresponding author:

V. Palanisamy (vpsgce@gmail.com) 
Huang S H [2] proved that Competition and the drive for profits are forcing companies to implement various productivity improvement efforts. Implementation of total productive maintenance (TPM) techniques has led to significant productivity improvements for individual equipment, particularly in the semiconductor industry. The productivity improvements achieved at the equipment level are significant but insufficient because what a company really needs is a highly efficient system/factory. This is especially true in the discrete manufacturing industry. In this paper, an approach, based on overall equipment effectiveness (OEE), is developed to model the productivity of a manufacturing system in terms of overall throughput effectiveness (OTE). Sensitivity analysis and theory of constraints are used to help identify productivity improvement opportunities. A real-world case study is presented to illustrate the applicability of the approach.

Remarkable improvements have occurred recently in the maintenance management of physical assets and productive systems, so that less wastage of energy and resources occur. The requirement for optimal preventive maintenance, using, for instance, just-in-time (JIT) and total quality-management (TQM) techniques, has given rise to what has been called the total productivemaintenance (TPM) approach. The study of Probert [3] explores the ways in which Nigerian manufacturing industries can implement TPM as a strategy and culture for improving its performance and suggests self-auditing and bench-marking as desirable prerequisites before TPM implementation.

Mark [4] put a strategy stating that developing society needs to adapt to change and foster creativity. In the pursuit of continual improvement (e.g., reducing fossilfuel consumption and waste, better service performance, greater availability and improved reliability), implementing wise maintenance-schedules is essential for contemporary organizations. Several studies of a wide range of Nigerian industries indicate that indigenous low availability and low productivity are endemic. The resulting closure of some of these industries has triggered off a realization of the strategic challenges in maintenance management. In addition, the increasingly-competitive business environment in Nigeria has raised the strategic importance of maintenance functions, especially in organizations with significant investments in physical assets. Five strategic aspects of maintenance management have been identified, namely: maintenance methodology; support processes; organization and work structuring; comparable culture; and general management policy [5-8]. Three factors that permeate these dimensions are wise leadership, excellent communications and an understanding of the human factors involved.

\section{Problem Definition}

There are a number of factors to improve productivity improvement in auto ancillary industry and prime areas to be focused are number of products to be produced in stipulated time in order to meet the customer demand, identification of minor defects, process leaks and other parameters related to machine defects. Unplanned downtime plays a vital role. The causes for this include improper maintenance, lack of skilled workers and movement of semi-finished parts from one place to another. In the Ergonomic point of view the employee should be in a position to access all the parts as feasible as possible. More factory floor space is utilized. Material handling equipment find difficulty in movement of semi-finished parts from one layout to another owing to rough and uneven floor space existing in the factory floor.

\section{Methodology}

Detailed Techniques about the Process

\subsection{Raw Materials}

- $\mathrm{ADC} 10$

- $\mathrm{ADC} 12$

- LM4

- LM6

\subsection{Inventory (Storage)}

Inventory is a storage unit of plant. In this plant they are using FIFO Technology (First In First Out) to provide the batch codes for the material and are stored in the inventory on the basis of customer wise separately.

\subsection{Material Handling System}

- STAGGER

- CRANE

- BIN \& TROLLEY 


\subsection{Manufacturing Process}

\section{STEP: 1 (Melting Process)}

This process is carried out in furnace. The raw materials are brought from the inventory into the factory for melting process. There are two different types furnace for a machine: Melting Furnace and Holding Furnace.

Both furnaces have the capacity of holding $300 \mathrm{~kg}$ of aluminum ore and having same electricity consumption of $36 \mathrm{kw}$. The temperature inside the furnace is from \pm 20 to $660^{\circ} \mathrm{C}$. The melting furnace has $50^{\circ} \mathrm{C}$ temp more than the holding furnace.

Material mixing ratio is $60: 40$ where, $60 \%$ of fresh bar and $40 \%$ of scrap. The temperature inside the furnace exceeds or decreed over the limit. The warning alarm automatically starts and cuts of the power supply.

\section{STEP: 2 (Material Cleaning)}

The raw materials are heated inside the furnace till $660^{\circ} \mathrm{C}$ at this stage a degassing tablet is added to the material. Degassing tablet is combination of chemical in the form of tablet which is used to dissolve hydrogen by diffusion process (For $300 \mathrm{~kg}$ of material $100 \mathrm{grms}$ degassing tablet is used). Immediately a coverall powder is mixed with the molten metal inside the furnace (For $300 \mathrm{~kg}$ of material $500 \mathrm{grms}$ of powder is used). This process is done continuously for three shifts per day. The dust and waste materials separated by the degassing tablet is brought to the top of the furnace by coverall powder. The dust particles are removed using a ladle. The slag taken out is stored in a barrel.

\section{STEP: 3 (Die - Casting Process)}

Die casting is a metal casting process that is characterized by forcing molten metal under high pressure into a mould cavity. The mold cavity is created using two hardened tool which have been machined into shape and work similarly to an injection mold during the process. Most die castings are made from non-ferrous metals, specifically zinc, copper, aluminum, magnesium, lead, pewter and tin based alloys. Depend on the type of metal being casted. In this process the molten material is taken from the furnace by a robotic arm to a sleeve. In sleeve hot plunger is forced to give pressure to the sleeve. After that hot dies are formed according to our required shapes. After forming the shape the ejectors are used to eject the material out by means of robotic arms.
- Die Coating

Die coating is a process which is done in between die casting process. This is done to prevent alloy getting attached to the die. Die coating is a mixture of Die coat oil and water forced by using pressurized air into the die

\section{- Cooling Tower}

Cooling tower is used to control the heat pressure formed inside a die casting machine and in the furnace. The water is passed into machine which converts water into steam and returns to the cooling tower where it transforms to water. This process is done continuously. While casting the hydraulic oil temperature may increase and the viscosity is decrease, in order to avoid that this process introduced to reduce the hydraulic oil temperature.

\section{STEP: 4 (In-Process Inspection)}

In-process inspection is done manually by workers to adjust the dies in correct positions they verifies the first product as per the drawing by using vernier height gauge and vernier caliber. The rejected pieces are sending back to furnace for re modeling.

\section{STEP: 5 (Fettling)}

The removal of feeders and excess material from a casting is the first stage of finishing a casting. The metal removal is often achieved using manual cutting or grinding. However, more emphasis is being placed on automatic fettling, whereby the casting is placed in a machine programmed to remove materials from specific areas. The method of fettling must be taken into account at the initial casting design stage, so that the process is fast and efficient.

\section{STEP: 6 (Shot Blasting)}

Blasting is a process of shooting abrasive particles, such as sand, grit or steel shot, are propelled at high velocity to impact the casting surface to remove surface contaminants. It can also be used to provide a uniform surface finish to castings. Propulsion of the abrasive particles is usually achieved using a centrifuge or compressed air nozzles.

\section{STEP: 7 (Final Inspection)}

After finishing, castings are inspected for surface quality. Inspection can be performed manually by visual checking, manually by template comparison, or by an automated inspection station. Visual inspection is simple yet 
informative. A visual inspection would include significant dimensional measurements as well as general appearance. In final pieces are sent back for minor reworks if any rejection is there it is sent back to the furnace.

\section{STEP: 7 (Packing)}

The final products are packed as per the requirements of the customers and are sent to the dispatching unit.

\section{OEE Calculations and its Parameters}

\subsection{Calculation Formula for OEE}

OEE $=$ Availability $\times$ Performance $\times$ Quality

\subsubsection{Availability}

The Availability portion of the OEE Metric represents the percentage of scheduled time that the operation is available to operate. The Availability Metric is a pure measurement of Uptime that is designed to exclude the effects of Quality, Performance, and Scheduled Downtime Events.

Availability $=$ Operating Time $/$ Planned Production Time

\subsubsection{Performance}

The Performance portion of the OEE Metric represents the speed at which the Work Center runs as a percentage of its designed speed. The Performance Metric is a pure measurement of speed that is designed to exclude the effects of Quality and Availability

Performance $=$ Ideal Cycle Time / (Operating Time / Total Pieces)

\subsubsection{Quality}

The Quality portion of the OEE Metric represents the Good Units produced as a percentage of the Total Units Started. The Quality Metric is a pure measurement of Process Yield that is designed to exclude the effects of Availability and Performance.

Quality $=$ Good Pieces / Total Pieces

\subsection{Existing OEE in the Job Floor}

\subsubsection{Production Data}

Shift length: 259200 Sec

Down time: $111900 \mathrm{Sec}$
Ideal cycle time: $30 \mathrm{Sec}$ Per Piece

Total piece: 3126

Reject piece: 147

\subsubsection{Support Variable}

Planned production $=259200 \mathrm{sec}$

Operating time $=259200-111900=147300 \mathrm{sec}$

Good piece $=3126-147=2979$

\subsection{OEE Factors}

Availability: $147300 / 259200=0.5682$

Performance: $3126 /(147300 / 30)=0.6549$

Quality: $2979 / 3126=0.9529$

$\mathrm{OEE}=(0.5682 \times 0.6549 \times 0.9529) \times 100=\mathbf{3 5 . 4 \%}$

The above calculation is based on the data for a single component that is manufacture in the job floor.

\subsection{Practices to Improve OEE in Job Floor}

- Introduced Cast iron thermocouple inside the crucible of the furnace for control the Furnace.

- Preventive maintenance is used to reduce the down time.

- Skilled persons are used to minimize reduced speed \& small stops.

\subsubsection{Other Suggestions}

- SMED (single minute exchange of Die) to be implemented in order to reduce the setup time of the machine.

- Bottleneck technique can be reduced by using best flooring techniques.

- EOT can be used to move die \& materials inside the company.

- 3C method can be used to improve the customer satisfaction.

- Labours training can be given by the skilled labours in the company.

\subsection{Improved OEE Calculation}

The above mentioned technique was practiced in the job floor and considerably the downtime was reduced from $111900 \mathrm{sec}$ to $105900 \mathrm{sec}$. This huge difference in downtime reduction say $(6000 \mathrm{sec})$ had a great impact in the production floor data and thus increased the productivity, effective utilization of the factory floor space and optimized the production data without any interference from the above said bottle neck operations. 


\subsubsection{Production Data}

Shift length: $259200 \mathrm{Sec}$

Down time: $105900 \mathrm{Sec}$

Ideal cycle time: $30 \mathrm{Sec}$ per Piece

Total piece: 4043

Reject piece: 173

\subsubsection{Support Variable}

Planned production $=259200 \mathrm{sec}$

Operating time $=259200-105900=153300 \mathrm{sec}$

Good piece $=4043-173=3870$

\subsection{OEE Factors}

Availability: $153300 / 259200=0.5914$

Performance: $4043 /(153300 / 30)=0.7911$

Quality: $3870 / 4043=0.9572$

$\mathrm{OEE}=(0.5914 \times 0.7911 \times 0.9572) \times 100=\mathbf{4 4 . 6 \%}$

\section{Conclusion}

OEE is not the solution to all the problems, in fact simply measuring OEE is of no use. OEE works best when it is used as a strategy i.e. as a part of overall organization improvement plan. Any policy deployment should cascade relevant elements of OEE data down to individual department managers and staff. This is necessary to ensure that it is not the sole responsibility of production or maintenance department to improve OEE; other departments are also equally involved.

OEE is a measure of overall efficiency of an organization and it has a direct impact on company's bottom line. A greater return on investment is expected by improving OEE. It is also an effective tool of comparison within plant, at different sites and also against competitors.

\section{Results and Discussion}

Skills matrix was developed aligned to the One Best Way, which highlighted key skills gaps across the organization. This was used to priorities training and coaching plans, at both an Individual and team level. The skills matrix also enabled experts to be identified who subsequently played a key role in developing other staff through training and coaching. Buddies were also identified to cover unplanned absence. Annual business objectives were revised for Project Managers, increasing the focus of the PMs and their line management on financial control during reviews.

\section{Scope of Future Work}

The data's obtained from the industry can be entered in the software and the simulated outputs can be interoperated. The software includes TIMERPRO and VIP-PLANOPT. The various parameters involved in causing the downtime should be periodically monitored and other quality tools should be implemented in the factory floor in order to improve the machine performance.

\section{References}

1. Wang T, and Pan H (2011). Improving the OEE and UPH data quality by Automated Data Collection for the semiconductor assembly industry, Expert Systems with Applications, vol 38(5), 5764-5773.

2. Huang S H (2004). Applied Energy, vol 79(4), 385-401.

3. Probert S D (2005). Implementing total productive maintenance in Nigerian manufacturing industries, International Journal of Production Economics, vol 95(1), 71-94.

4. Mark C (2005). Applied Energy, vol 82(3), 255-265.

5. Blanchard B S (1997). An enhanced approach for implementing total productive maintenance in the manufacturing environment, Journal of Quality in Maintenance Engineering, vol 3(2), 69-80.

6. Chan F T S et al. (2005). Implementation of total productive maintenance: a case study, International Journal of Production Economics, vol 95, 71-94.

7. Eti M C et al. (2004). Implementing total productive maintenance in Nigerian manufacturing industries, Applied Energy, vol 79(4), 385-401.

8. Hartmann E H, and Charles H L (2001). Total productive maintenance, in Maynard's Industrial Engineering Handbook, Zandin K B (Ed.), McGraw-Hill, New York, 16.57-16.77. 